\title{
A Computational Framework for Automation of Point Defect Calculations
}

\author{
Anuj Goyal ${ }^{\mathrm{a}, \mathrm{b}}$, Prashun Gorai ${ }^{\mathrm{a}, \mathrm{b}}$, Haowei Peng ${ }^{\mathrm{b}}$, Stephan Lany ${ }^{\mathrm{b}}$, Vladan Stevanovića ${ }^{\mathrm{a}, \mathrm{b}}$ \\ ${ }^{a}$ Colorado School of Mines, Golden, CO 80401 USA \\ ${ }^{b}$ National Renewable Energy Laboratory, Golden, CO 80401, USA
}

\begin{abstract}
A complete and rigorously validated open-source Python framework to automate point defect calculations using density functional theory has been developed. The framework provides an effective and efficient method for defect structure generation, and creation of simple yet customizable workflows to analyze defect calculations. The package provides the capability to compute widely-accepted correction schemes to overcome finite-size effects, including (1) potential alignment, (2) image-charge correction, and (3) band filling correction to shallow defects. Using $\mathrm{Si}, \mathrm{ZnO}$ and $\mathrm{In}_{2} \mathrm{O}_{3}$ as test examples, we demonstrate the package capabilities and validate the methodology.
\end{abstract}

Keywords: Point defects, High-throughput, Density-functional theory, Finite-size corrections, Materials Genome Initiative

\section{Introduction}

In semiconductor materials, point defects play a vital role in determining their properties and performance, particularly in microelectronics [1], optoelectronics [2], and thermoelectrics [3] related applications. The dominant point defects and their concentrations are determined from the defect formation energies, which can be predicted with reasonable accuracy [4] using first-principles methods such as density functional theory (DFT). Therefore, computational modeling of point defects is increasingly becoming an indispensable tool to understand and predict behavior of semiconductors [5, 6, 7]. Modern approaches to point defect calculations uses DFT and are typically based on the supercell approach $[6,7]$. With the goal of accelerating the design and discovery of materials by large-scale deployment of defect calculations, we have developed a computational framework (Fig. 1)

Email addresses: anuj.goyal@nrel.gov (Anuj Goyal), Vladan.Stevanovic@nrel.gov (Vladan Stevanović)

Preprint submitted to Computational Materials Science

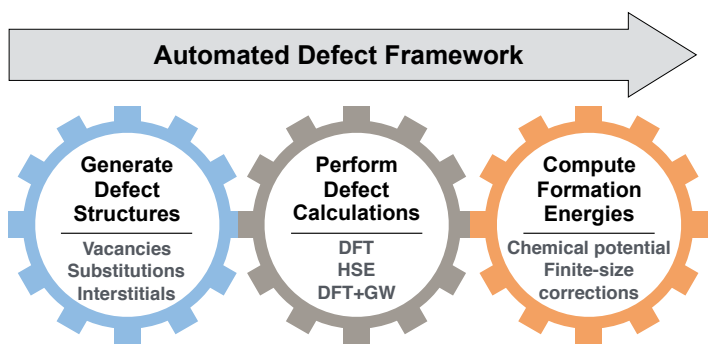

Figure 1: Three key components of the computational framework to automate point defect calculations.

to automate supercell-based point defect calculations with DFT. Our approach successfully addresses two main challenges of automating point defect calculations: (1) generation of defects structures including vacancies, substitutional defects and interstitials, and (2) application of the finite-size and band gap corrections.

In the context of structure generation, creating supercells with vacancies and substitutional defects is relatively straightforward. In contrast, identify-

(C) 2017. This manuscript version is made available under the Elsevier user license http://www.elsevier.com/open-access/userlicense/1.0/ 
ing likely locations of interstitials is much more challenging because of the large number of interstitialcy sites, especially in complex, multinary systems. In addition, interstitials might adopt complex configurations, including the split or dumbbell where the interstitial is associated with a off-site lattice atom. To address these challenges, we have developed an efficient scheme based on Voronoi tessellation; [8] the scheme considers corners, edge and face centers of the Voronoi polyhedra as likely sites for interstitials. We demonstrate that, upon relaxing the structure, this scheme successfully discovers both the symmetric and general Wyckoff positions as well as the split interstitial configurations. Our implementation of this scheme is independent of pymatgen [9] where Voronoi tessellation is also employed. Here we will discuss the algorithm in detail and validate the Voronoi-driven approach to identify interstitial sites.

Within the supercell approach to calculate the defect formation energies, finite-size artifacts need to be removed using carefully designed correction schemes. We have implemented tools to calculate the following finite-size corrections: (1) potential alignment, (2) image-charge correction, and (3) band filling correction to address Moss-Burstein-type effects. We follow the widely used and tested approach of Lany and Zunger $[10,11]$ out of the several others that addresses the same issues $[12,13,14,15,16]$. However, the automated framework is highly modular so that other correction schemes can be easily implemented including computation of defect formation energies using series of supercell sizes in order to extrapolate the values to the infinitely large supercell. In addition, the framework employs fitted elemental-phase reference energies (FERE) [17, 18] to compute elemental chemical potentials, which have been shown to provide accurate predictions of thermodynamic phase stability.

Beyond the finite-size effects, another source of inaccuracy arises from the well-known DFT band gap problem. Accurate band gaps are needed to correctly describe the formation energy of charged defects as a function of the electronic chemical potential i.e., Fermi energy. We employ state-of-the-art GW quasiparticle energy calculations [19] to compute band edge shifts (relative to the DFT-computed band edges). The band edge shifts are used to correct the defect formation energy in multiple charge states. The automated framework is also capable of performing defect calculations with DFT hybrid functionals $[20,21]$. However, supercell defects calculations with hybrid functionals have sources of uncertainty arising from the choice of input parameters (e.g. fraction of exchange) and have considerable computational overheads [22]. Therefore, we have implemented a $\mathrm{DFT}+\mathrm{GW}$ approach for calculating defect formation energy that has been shown to be as accurate as calculations with hybrid functionals [22].

Finally, we illustrate and validate the automated computational framework by considering the set of three well-studied semiconductor materials, $\mathrm{Si}, \mathrm{ZnO}$, and $\mathrm{In}_{2} \mathrm{O}_{3}$ with a total of 17 unique interstitial and vacancy structures in multiple charged states. We show that our results on defect formation energies and charge defect transition levels in $\mathrm{Si}, \mathrm{ZnO}$ and $\mathrm{In}_{2} \mathrm{O}_{3}$ agree well with the literature. The framework successfully identifies the known intrinsic interstitial and vacancy structures in $\mathrm{Si}, \mathrm{ZnO}$ and $\mathrm{In}_{2} \mathrm{O}_{3}$. In addition, it discovers interstitial structures in $\mathrm{In}_{2} \mathrm{O}_{3}$, with formation energies $\sim 0.5 \mathrm{eV}$ above that of previously known interstitial structures.

\section{Overview of the Automated Defect Frame- work}

Figure 2(a) presents a workflow of the automated framework, including generation of defect structures, relaxation of defect supercells within DFT using the PyLada framework [23], and determination of finitesize and band gap corrections to compute the defect formation energies. In this section, we describe each component of the framework and provided technical details. The latest version of the package can be downloaded from GitHub repository at https: //github.com/pylada/pylada-defects.

\subsection{Generate Defect Structures}

The workflow takes the fully-relaxed primitive cell as an input to create supercells. To create a vacancy or substitutional defect in supercell, the occupied Wyckoff positions (lattice-sites) for all atom 
(a)

(b)

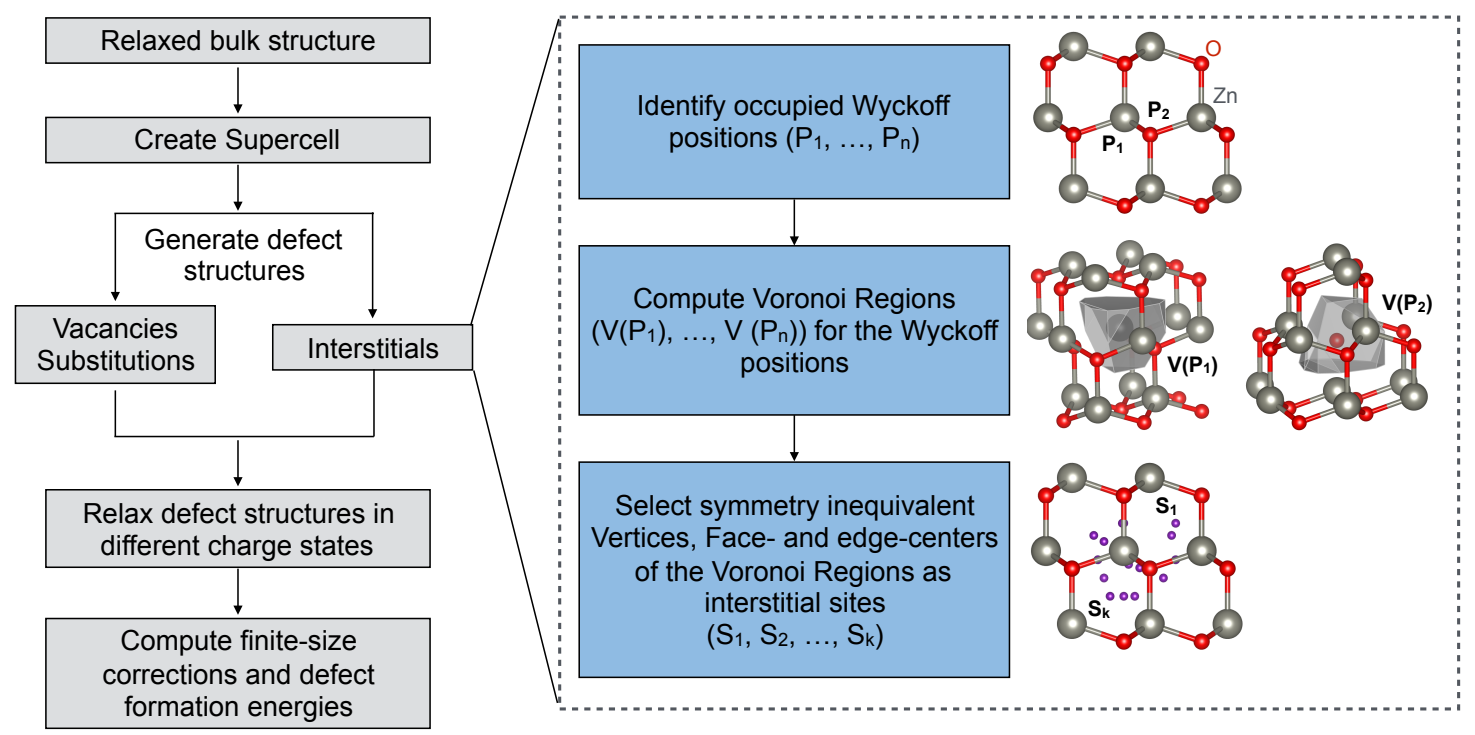

Figure 2: (a) Workflow to perform defect calculations, and (b) the Voronoi tessellation-based algorithm to find interstitial sites in a given structure (example shown: $\mathrm{ZnO}$ ).

types in the supercell are identified. Then the corresponding atom is removed or substituted with an impurity atom, to generate vacancy or substitutional defect. Finally, the first nearest-neighbor atoms to the vacancy or substitutional site are randomly displaced $(\sim 0.1 \AA)$ to break the underlying site symmetry and thereby, ensuring the non-symmetric configurations of the defects are properly captured. The Voronoi tesselation [8, 24], scheme is employed to identify likely interstitial sites. Voronoi region is the volume that encloses the points $p$ closest to a given lattice site $P_{i}$ than to any other lattice site $P_{j}$ for $i, j \in I_{n}=\{1, \ldots, n\}$. Mathematically, it is defined as $[8]$

$$
V\left(P_{i}\right)=\left\{p \mid d\left(p, P_{i}\right) \leq d\left(p, P_{j}\right)\right\} \text { for } j \neq i, j \in I_{n}
$$

where, $V\left(P_{i}\right)$ is the Voronoi region associated with $P_{i}$, and $d\left(p, P_{i}\right)$ specifies the minimum distance between a general point $p$ and $P_{i}$. To create an interstitial, Voronoi regions (Eq. 1) are computed across each occupied Wyckoff positions, and symmetry inequiv- alent vertices, face, and edge centers of the Voronoi regions are chosen as the candidate sites for the interstitials. The number of candidate interstitial sites depends on the symmetry of the crystal structure. The lower the symmetry and the more complex the crystal structure, the larger the number of sites. For example, in $\mathrm{In}_{2} \mathrm{O}_{3}$ (space group Ia-3, 40 atoms in primitive cell), we find that some of the faces of the Voronoi region are very small, resulting in sampled interstitial sites very close to each other. Therefore a minimum tolerance of $0.5 \AA$ is used while determining symmetry inequivalent sites. The procedure is described in Fig. 2(b), with $\mathrm{ZnO}$ as an example structure.

\subsection{Perform Defect Calculations}

As summarized in Fig. 2(a), the workflow starts with fully relaxing (volume, cell shape and ionic positions) the bulk primitive cell. Dielectric constant, and GW calculations are performed on the relaxed primitive cell. Point defects are then created in the bulk supercell followed by relaxation (only ionic positions) of defect structures in multiple charge states. 
Defect calculations are aimed to use supercell sizes that are large enough to describe individual defects as accurately as possible by minimizing the error due to defect-defect interactions. It has been shown that these interactions are short-ranged and typically occur within the distance of 5-10 $\AA$, from the defect center [7]. Therefore in this work, supercell sizes are chosen such that spacing between defect and its periodic images are greater than $10 \AA$, and sizes are not too large to make them intractable for DFT calculations. Calculations of interstitial defects are performed in two steps: (1) All candidate interstitials (shown as starting interstitials in Fig. 3) are relaxed in the neutral charge state, (2) followed by relaxation of only unique interstitials (shown as final interstitials in Fig. 3) in multiple charge states. Unique interstitial structures are determined based on: (1) the total energy, (2) space group, and (3) the number of neighboring atoms. The high-throughput DFT calculations are performed using PyLada [23], a Python framework for the organizing and managing highthroughput first-principles calculations. PyLada also offers variety of useful tools for manipulating crystal structures, extracting output from successfully finishes calculations, as well as archiving and analyzing results [25, 26, 27]. Finally, the defect formation energies are computed as discussed in the next section.

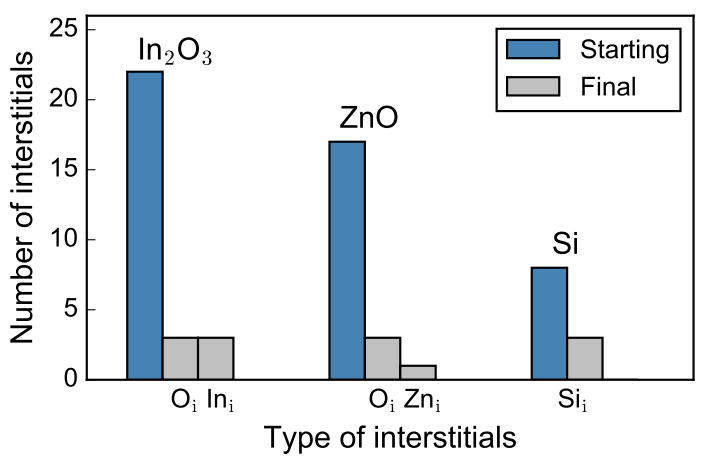

Figure 3: Number of distinct starting (blue) and final DFT relaxed (silver) interstitial structures in $\mathrm{Si}, \mathrm{ZnO}$, and $\mathrm{In}_{2} \mathrm{O}_{3}$.

\subsection{Compute Defect Formation Energy}

The formation energy of the defect $\mathrm{D}$ in the charge state $q$ is calculated as

$$
\Delta H_{\mathrm{D}, \mathrm{q}}\left(E_{F}, \mu\right)=\left[E_{\mathrm{D}, \mathrm{q}}-E_{\mathrm{H}}\right]+\sum_{i} n_{i} \mu_{i}+q E_{F}+E_{\mathrm{corr}}
$$

where, $E_{\mathrm{D}, \mathrm{q}}$ and $E_{\mathrm{H}}$ are the total DFT energies of the defect and host supercell, respectively. $\mu_{i}$ is the chemical potential of the atom (host or impurity) of type $i$ added $\left(n_{i}<0\right)$ or removed $\left(n_{i}>0\right)$ from the host supercell to form the defect. $E_{F}$ is the Fermi energy, and $E_{\text {corr }}$ is the term that account for the finite-size corrections, within the supercell approach. A schematic of Eq. 2, representing computation of the defect formation energy from supercell to the dilute limit is shown in Fig. 4.

\subsubsection{Chemical Potential and Phase Stability}

Chemical potentials $\mu_{i}$ reflect the energy of the reservoirs for the atoms that are involved in creating the defect. Numerical values of the chemical potentials $\left(\mu_{i}=\mu_{i}^{0}+\Delta \mu_{i}\right)$ depend on their implicit references, $\mu_{i}^{0}$, which here are obtained from the reference FERE $[17,18]$ energies, $\mu_{i}^{0}=\mu_{i}^{\mathrm{FERE}}$. FERE energies are also used to compute the formation enthalpy $\left(\Delta H_{f}\right)$ of all the competing phases which are needed to determine the thermodynamic limits of the chemical potential $\Delta \mu_{i}$. Following the FERE approach [18] the framework provides the necessary results to determine the valid range of chemical potentials, and plotting the final results of defect energy versus chemical potential is left to the user. The computed $\Delta H_{f}$ and $\mu_{i}^{\mathrm{FERE}}$ values are summarized in table 1 . For $\mathrm{Si}$, $\mu_{S i}^{0}=E^{\mathrm{GGA}}(\mathrm{Si})=-5.41 \mathrm{eV} /$ atom is used and to determine the limits to the respective elemental chemical potentials, we apply the following thermodynamic stability conditions, $\Delta \mu_{\mathrm{Zn}}+\Delta \mu_{\mathrm{O}}=\Delta H_{f}(\mathrm{ZnO})$ and $2 \Delta \mu_{\mathrm{In}}+3 \Delta \mu_{\mathrm{O}}=\Delta H_{f}\left(\mathrm{In}_{2} \mathrm{O}_{3}\right)$, in $\mathrm{ZnO}$ and $\mathrm{In}_{2} \mathrm{O}_{3}$, respectively.

\subsubsection{Electron Chemical Potential}

Fermi energy is the measure of the chemical potential of electrons. It is defined with respect to the host valence band maximum (VBM), $E_{F}=E_{\mathrm{VBM}}^{\mathrm{Host}}+\Delta E_{F}$, 


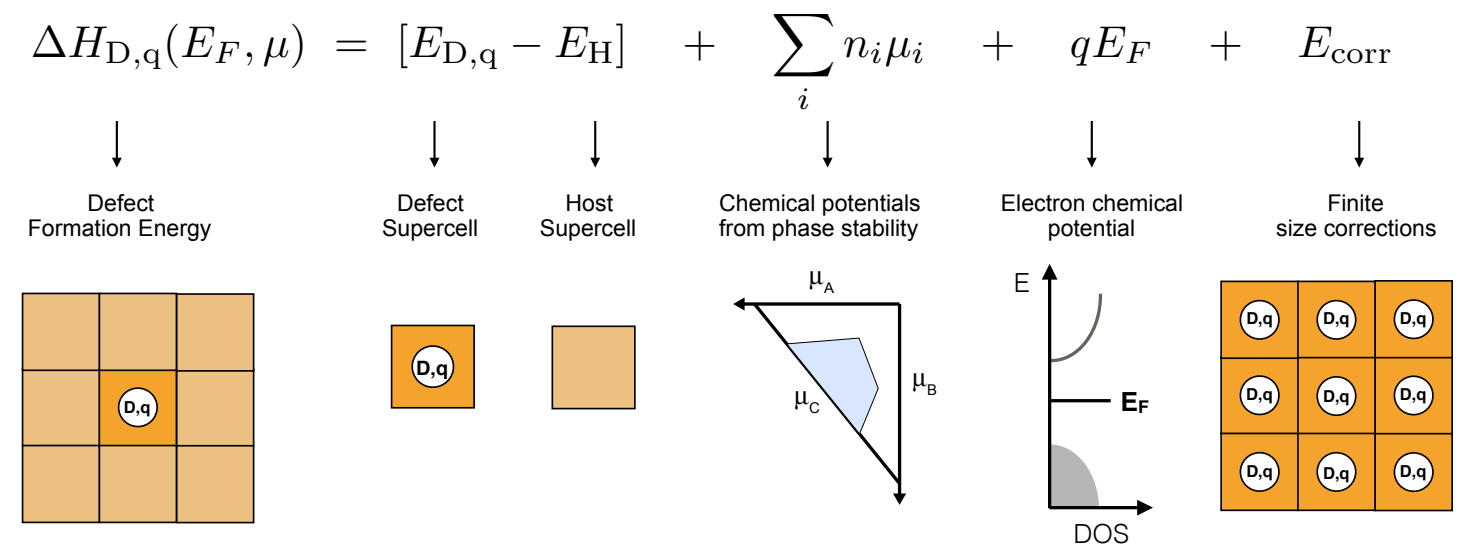

Figure 4: Equation to compute charge defect formation energy as function of the chemical potential and Fermi energy.

and is bounded by the conduction band maximum (CBM). DFT (GGA) band gaps are corrected by determining the band edge shifts, $\Delta E_{V}$ for the VBM, and $\Delta E_{C}$ for the CBM, from the GW quasiparticle energy calculations [22]. The computed band gaps are also summarized in table 1.

\subsubsection{Finite-size Corrections}

Finite size corrections are implemented in the package following the approach of Lany and Zunger $[10,11]$. Correction schemes focusing on single physical effect are considered [7]. These include:

Potential alignment correction, which restores the relative position of the host VBM in the calculations of charged defect (affecting the Fermi energy). Correction to the defect formation energy due to the potential alignment is given as [10]

$$
E_{\mathrm{PA}}(\mathrm{D}, \mathrm{q})=\mathrm{q}\left(V_{\mathrm{D}, \mathrm{q}}^{r}-V_{\mathrm{H}}^{r}\right)
$$

where the reference potentials, $V^{r}$, for the charged defect $(\mathrm{D}, \mathrm{q})$ and the pure host $(\mathrm{H})$ are determined from the (local) atomic-sphere-averaged electrostatic potentials at the atomic sites farther away from the defect [10].

Image-charge correction, is needed to correct for the spurious electrostatic interactions of the charged defect (in the presence of homogeneous compensating background charge) with its periodic images. This is given as [11]

$$
E_{\mathrm{IC}}=\left[1+c_{s h}\left(1-\frac{1}{\varepsilon}\right)\right] \frac{q^{2} \alpha_{M}}{2 \varepsilon L}
$$

where $L=\Omega^{-1 / 3}$ is the linear supercell dimension (volume, $\Omega$ ), $\varepsilon$ is the static dielectric constant (electronic + ionic), and $\alpha_{M}, c_{s h}$ are the Madelung constant, and shape factor, respectively, for the adopted supercell geometry.

Band filling correction, correct for the MossBurstein-type band filling effects that appear due to high defect concentrations in a typical finite-size supercell calculations [10]. For a given k-point set (weighted sum, $w_{k}$ ) and band occupations, $\eta_{n, k}$, the correction for the shallow donor is computed as [10]

$$
E_{\mathrm{BF}}=-\sum_{n, k} w_{k} \eta_{n, k}\left[e_{n . k}-\tilde{e_{C}}\right]
$$

and for shallow acceptor

$$
E_{\mathrm{BF}}=\sum_{n, k} w_{k}\left(1-\eta_{n, k}\right)\left[e_{n . k}-\tilde{e_{V}}\right]
$$

where, $e_{n . k}$ are the band energies in the defect calculation, $\tilde{e_{C}}$ is the CBM and $\tilde{e_{V}}$ is the VBM energy of the pure host after potential alignment correction. 
Table 1: Calculated lattice parameters, dielectric constants (electronic, $\varepsilon_{\text {elec. }}$, and ionic $\varepsilon_{\text {ionic }}$ ), chemical potential, enthalpy of formation and band gap in $\mathrm{Si}(\mathrm{Fd}-3 \mathrm{~m}, 227)$, $\mathrm{ZnO}\left(\mathrm{P} 6_{3} \mathrm{mc}, 186\right)$ and $\mathrm{In}_{2} \mathrm{O}_{3}$ (Ia-3, 206). Experimental values are also cited.

\begin{tabular}{|c|c|c|c|c|c|c|}
\hline \multirow[t]{2}{*}{ System } & \multirow{2}{*}{$\begin{array}{l}\text { Lattice constant } \\
\qquad(\AA)\end{array}$} & \multicolumn{2}{|c|}{ Dielectric constant } & \multirow{2}{*}{$\begin{array}{c}\text { Chemical potential } \\
\mu_{i}^{\text {FERE }}(\mathrm{eV})\end{array}$} & \multirow{2}{*}{$\begin{array}{l}\Delta \mathrm{H}_{f} \\
(\mathrm{eV})\end{array}$} & \multirow{2}{*}{$\begin{array}{c}\text { Band Gap (eV) } \\
\text { GW (GGA) }\end{array}$} \\
\hline & & $\varepsilon_{\text {elec. }}$ & $\varepsilon_{\text {ionic }}$ & & & \\
\hline $\mathrm{Si}$ & 5.46 & 13.36 & & -5.41 & & $1.29(0.62)$ \\
\hline Expt. ${ }^{1}$ & 5.43 & 11.7 & & & & 1.17 \\
\hline $\mathrm{ZnO}$ & $\begin{array}{c}a=3.28 \\
c=5.30\end{array}$ & 5.53 & 5.12 & $\begin{array}{l}\mathrm{O}=-4.76 \\
\mathrm{Zn}=-0.56\end{array}$ & -3.63 & $3.25(0.73)$ \\
\hline Expt. ${ }^{2}$ & $a=3.25, c=5.2$ & $3.7-3.8$ & $4-5.13$ & & -3.62 & 3.44 \\
\hline $\mathrm{In}_{2} \mathrm{O}_{3}$ & 10.28 & 4.90 & 6.47 & $\begin{array}{l}\mathrm{O}=-4.76 \\
\mathrm{In}=-2.31\end{array}$ & -9.45 & $2.47(0.96)$ \\
\hline Expt. 3 & 10.1 & 4.08 & 4.8 & & -9.6 & $2.67-3.1$ \\
\hline & Split & (b) Tetrahed & & (c) Hexagonal & $\Rightarrow$ & Non-symmetric \\
\hline
\end{tabular}

Figure 5: Self interstitial structures in Si: (a) split, (b) tetrahedral, and (c) hexagonal geometry. Hexagonal interstitial has symmetric and non-symmetric ' $\mathrm{C}_{v}$ ' configuration as shown in the projection along [110] direction in the side view.

\section{Examples}

\subsection{Silicon}

Silicon has been the focus of both experimental $[41,42]$ and theoretical $[43,44,45,46,47,48,49]$ research on intrinsic point defects over the past decade. Structure of both silicon vacancies [43, 46, 47, 49] and self-interstitials [44, 45, 48] has been topic of interest as they exists in several stable and metastable configurations. DFT calculations are performed with the projector augmented wave (PAW) method [50] as implemented in VASP [51]. The Perdew Burke Ernzerhof (PBE) exchange correlation functional [52] is used in GGA spin-polarized calculations. A plane wave energy cutoff of $340 \mathrm{eV}$, and a Monkhorst-Pack k-point sampling [53] is used. The structures are taken from the Inorganic Crystal Structure Database (ICSD) [54] and fully relaxed using the procedure outlined in Ref. 18. Defect calculations are performed on 216 atoms supercell, as there is significant dispersion of defect levels in a smaller 64 atoms supercell, which lead to spurious results. A single $\Gamma$ point only calculations are performed. The low-frequency total (electronic + ionic) dielectric constant is obtained following the procedure in Ref. 22. For hybrid functional (HSE06 [55]) calculations, the exchange mixing, $\alpha=0.25$ is 


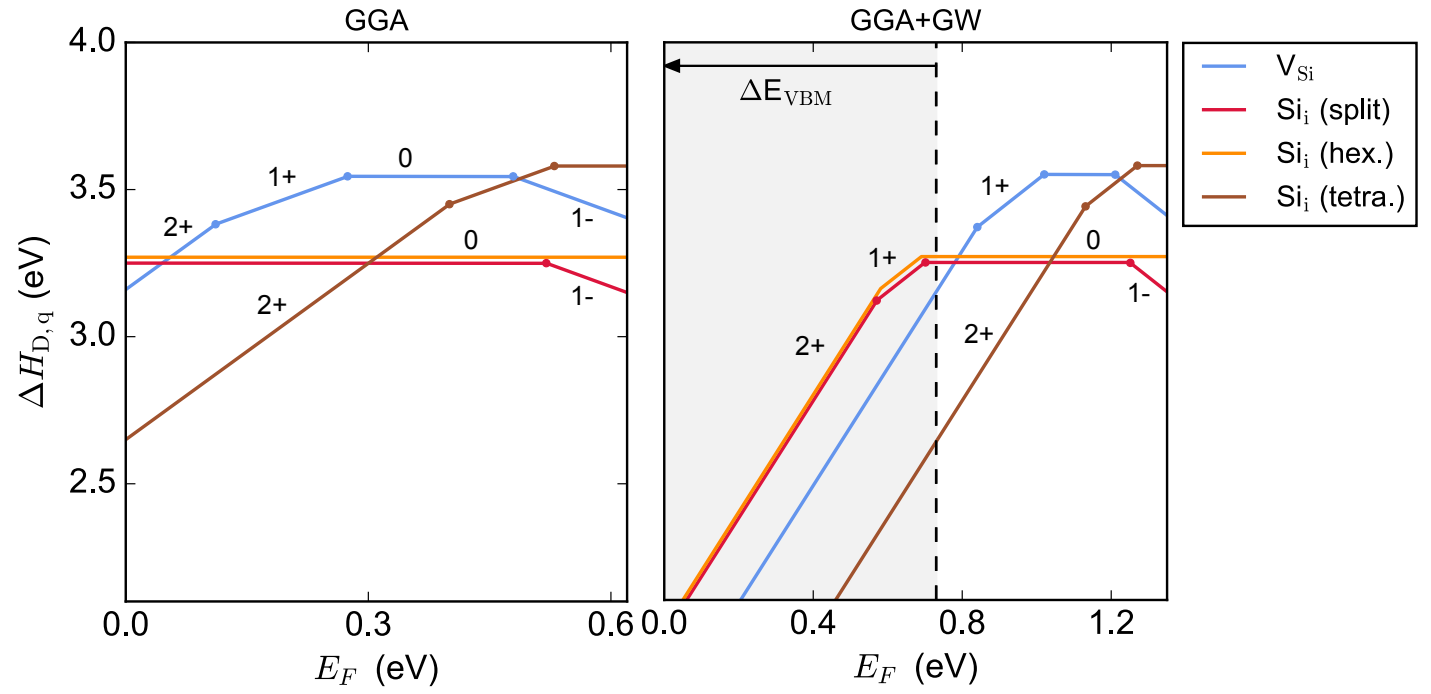

Figure 6: Defect formation energy as function of the Fermi energy from GGA and GGA+GW calculations.

used. GW calculations are performed on the DFT relaxed structures, with the unit cell vectors re-scaled to match the experimental lattice volume [22].

In agreement with the existing literature, we find three distinct silicon self-interstitial structures namely, spit, hexagonal and tetrahedral as shown in Fig. 5, among the starting 7 candidate sites from the defect generation code. Neutral split interstitial has the lowest formation energy $(3.25 \mathrm{eV})$, followed by hexagonal interstitial with energy $0.2 \mathrm{eV}$ higher. Hexagonal interstitial lies along the [111] direction and sit symmetrically at the center of the hexagon formed by six neighboring Si lattice atoms. Hexagonal interstitial also exists in a non-symmetric configuration, as shown in the side view in Fig. 5. This configuration is $5 \mathrm{meV}$ lower in energy than the symmetric one, but is unstable and relaxes to tetrahedral geometry in the charge states $1+$ and $2+$. Metastable hexagonal configuration have been reported in previous DFT calculations [48], referred as 'displaced hexagonal' or by $\mathrm{C}_{3 v}$ site symmetry. Tetrahedral interstitial in the neutral charge state has the highest formation energy, about $0.33 \mathrm{eV}$ higher than the split interstitial. All the distances between the interstitial and the four neighboring Si lattice sites are same and are equal to $2.47 \AA$.

Vacancy structures are analyzed in terms of distances between the neighboring 4 silicon atoms to the vacancy site. It has been reported [41] that silicon vacancy undergo structure reconstruction in different charge states, and form a Negative-U system $[43,41]$. The negative-U behavior implies an energy lowering structural distortion by the presence of a second electron, such that the energy gain more than compensates the $e$-e repulsive energy cost [43]. For the spin-polarized calculations, vacancy in the neutral and 2- charge state relaxes to higher energy configurations, with $\mathrm{C}_{2 v}$ and $\mathrm{D}_{2 d}$ point group symmetry, respectively. The inability of spin-polarized calculations to reproduce lower energy point group symmetry of charged vacancies has also been reported in previous LDA [56] and GGA [49] calculations. We observe $(2+/ 1+)$ and $(1+/ 0)$ charge transitions for silicon vacancy, instead of the direct $(2+/ 0)$ charge transition because the computed neutral Si vacancy is in the higher energy configuration compared to its lower energy $\mathrm{D}_{2 d}$ configuration. However, using spinpolarized HSE calculations on DFT structures, we observe direct $(2+/ 0)$ charge transition.

Among interstitials, tetrahedral structure is most 


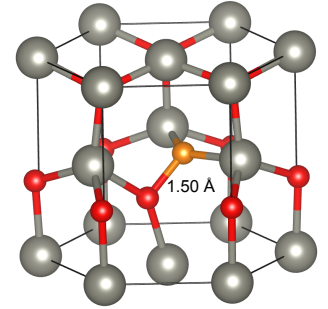

(a) Split

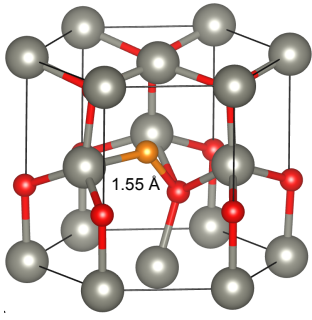

(b) Split*

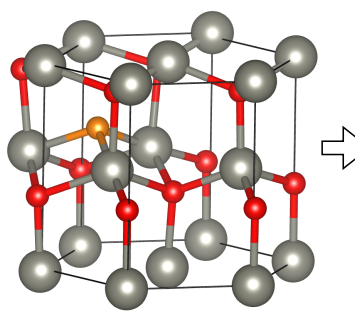

(c) Octahedral

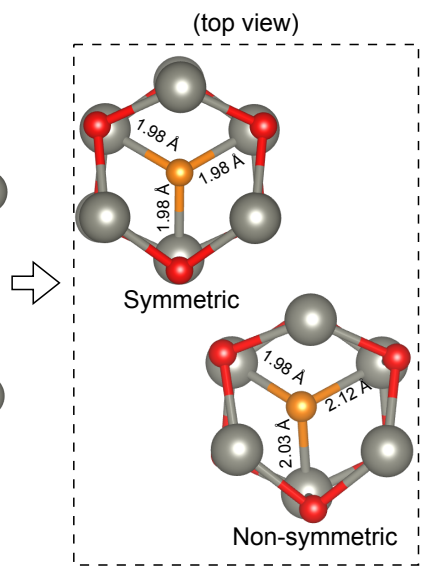

Figure 7: Oxygen interstitial structures (in orange) in ZnO: (a) split, (b) split*, and (c) octahedral geometry. Octahedral oxygen interstitial has symmetric and non-symmetric configuration as shown in projection along [001] Z-axis in the top view.

stable in $2+$ charge state, which then transition $(-0.27$ $\mathrm{eV}$ below $\mathrm{CBM}$ ) to the split structure, which is the most stable configuration for 0 and 1- charge states. The computed defect formation energies and charge transition levels (Fig. 6) for silicon vacancies and interstitials are in good agreement with the reported GGA $[48,56,49]$ and HSE calculations [57, 49]. However, their are noticeable difference in the charge defect transition levels between GGA and GGA+GW, mainly due to the band edge positions predicted by the self-consistent GW calculation [58]. Similar differences in charge transition levels between LDA and $\mathrm{LDA}+\mathrm{G}_{0} \mathrm{~W}_{0}$ in calculations on silicon interstitials has been reported by Rinke et al. [48]

\subsection{Zinc Oxide}

$\mathrm{ZnO}$ is a direct band gap semiconductor and occurs in the ground state wurtzite crystal structure (space group $\mathrm{P} 6_{3} \mathrm{mc}, 186$ ), with two lattice parameters, $a$ and $c$, in the ratio of $c / a=1.63$. Spinpolarized $\mathrm{GGA}+\mathrm{U}(\mathrm{U}(\mathrm{Zn}-\mathrm{d})=6 \mathrm{eV})$ calculations are performed on 96 atoms supercell, with A $\Gamma$-centered $2 \times 2 \times 2$ k-point mesh. The calculated lattice constants and band gap for the wurtzite $\mathrm{ZnO}$ are in good agreement with the known experimental measurements, as summarized in table 1. Comprehensive studies of intrinsic vacancy and interstitial structures $[59,60]$ in $\mathrm{ZnO}$ has been done in the past. In the following discussion we analyze the defect structures predicted using the automated defect framework and compare our results with the existing literature.

Figure 7 shows the obtained three distinct oxygen interstitial structures out of the starting 17 possibilities for the interstitial sites. Among oxygen interstitials, split interstitial (Fig. 7(a)) has the lowest formation energy. Split interstitial has a metastable configuration (Fig. 7(b), referred here, and in literature as split* [61, 59]) which is $0.21 \mathrm{eV}$ higher in energy than the stable split configuration and relaxes to stable configuration on further relaxation. Octahedral oxygen interstitial lies in the empty channel along [001] Z-axis inside the six member ring formed by $\mathrm{O}-\mathrm{Zn}$ atoms, Fig. $7(\mathrm{c})$, and is about $1.8 \mathrm{eV}$ higher in energy than the neutral split interstitial. We find two configurations of octahedral oxygen interstitial as shown in the projection along [001] Z-axis (dashed box) in Fig. 7. The symmetric configuration is 0.3 $\mathrm{eV}$ higher in energy than the non-symmetric configuration. The symmetric octahedral is only stable in the neutral charge state and relaxes to non-symmetric configuration for positive and negative charge states. The low energy non-symmetric octahedral configuration has been reported in a previous DFT study [60], investigating migration path of oxygen interstitials along [001] direction. With our method we directly find the non-symmetric configuration as the lowest 

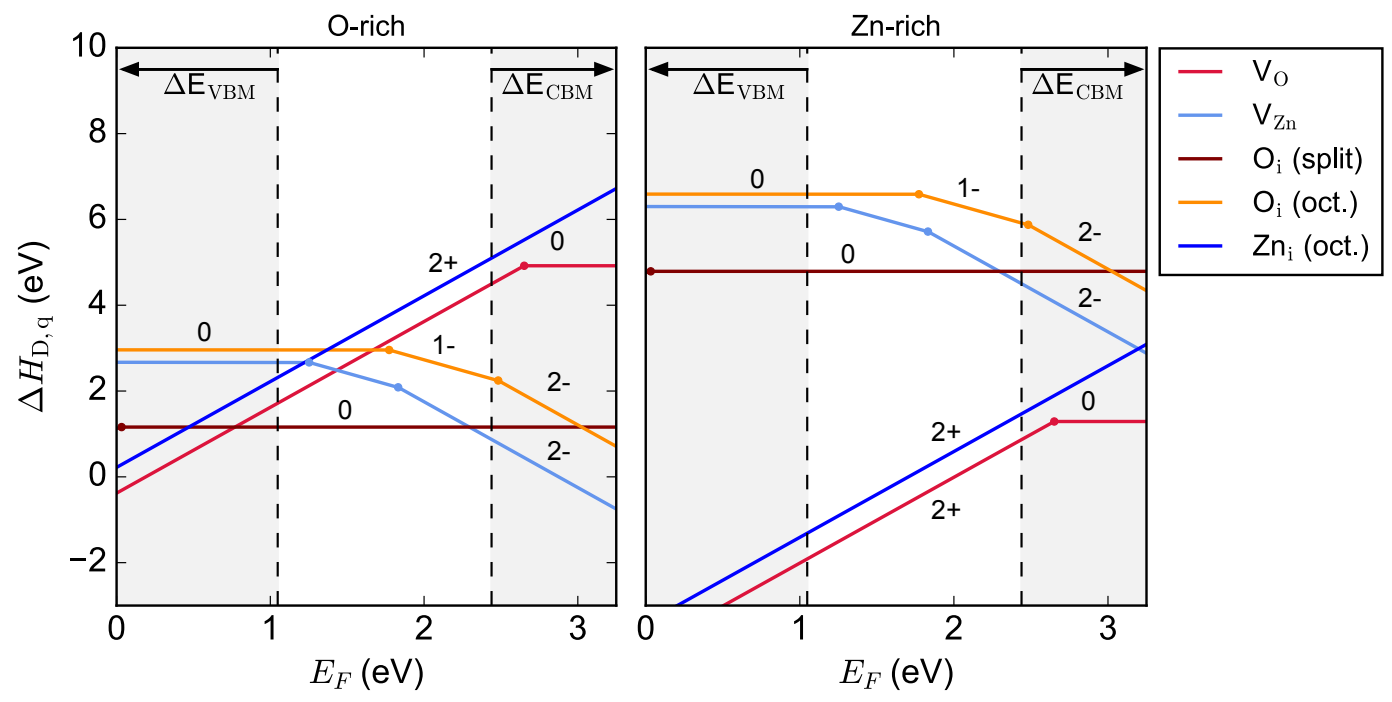

Figure 8: Defect formation energy as function of the Fermi energy for intrinsic vacancies and interstitials in ZnO using GGA+U with band edge shift computed from GW calculations.

energy octahedral structure.

Zinc interstitial is stable in the octahedral configuration with interstitial atom symmetrically placed at the center of the empty channel along [001] Z-axis, similar to the symmetric octahedral oxygen interstitial. In the relaxed geometry the $\mathrm{Zn}_{i}$-O distance is $2.05 \AA$, and $\mathrm{Zn}_{i}-\mathrm{Zn}$ distance is $2.45 \AA$. Among interstitials split oxygen interstitial is stable in neutral charge state for the whole range of Fermi Energy (Fig. 8). Oxygen interstitials at the octahedral site act as deep acceptors, and have relatively high formation energies compared to Zinc vacancies. Zinc interstitials act as shallow donors, with $2+$ charge as the most stable charge state (Fig. 8). But with formation energies as high as $2.6 \mathrm{eV}$ at CBM, even under $\mathrm{Zn}$ rich conditions are unlikely to form in substantial concentration.

Both oxygen and zinc in $\mathrm{ZnO}$ occupy the $2 \mathrm{~b}$ Wyckoff position, with 4-fold coordinated tetrahedral geometry. Oxygen vacancy in $2+$ charge state shows relatively large outward relaxation of the neighboring $\mathrm{Zn}$ atoms, as reported in previous DFT calculations [59]. Oxygen vacancy shows transition from $2+$ to 0 charge state, at Fermi energy $-0.45 \mathrm{eV}$ below the CBM (Fig. 8), confirming the reported Negative-U character $[59,62,63]$. Oxygen vacancy act a deep donor, with fully occupied neutral defect state inside the band gap. Zinc vacancy has partially occupied defect states in the band gap, and act as deep acceptor with (0/1-) and (1-/2-) transition level occur at 1.16 , and $1.58 \mathrm{eV}$, respectively above the VBM.

Overall, our approach confirms the known interstitial and vacancy structures in $\mathrm{ZnO}$, and provide a clear picture of the defect energetics and electronic structure consistent with the previous defect calculations. Our next step forward is to investigate the automated point defect framework against $\mathrm{In}_{2} \mathrm{O}_{3}$, a relatively complex crystal structure containing 40 atoms in the primitive unit cell.

\subsection{Indium Oxide}

$\mathrm{In}_{2} \mathrm{O}_{3}$ is a direct band gap semiconductor which is widely used as a transparent conducting oxide. Intrinsic defects in $\operatorname{In}_{2} \mathrm{O}_{3}$ have received relatively moderate attention both experimentally $[34,64]$ and theoretically $[62,65,66]$ compared to silicon and $\mathrm{ZnO}$. It crystalizes in ground state cubic bixbyite structure (space group Ia-3, 206) with indium (Wyckoff positions $8 \mathrm{~b}$ and $24 \mathrm{~d}$ ) and oxygen (48e) lattice sites in the bulk structure as shown in Fig. 9(a). Spin-polarized 


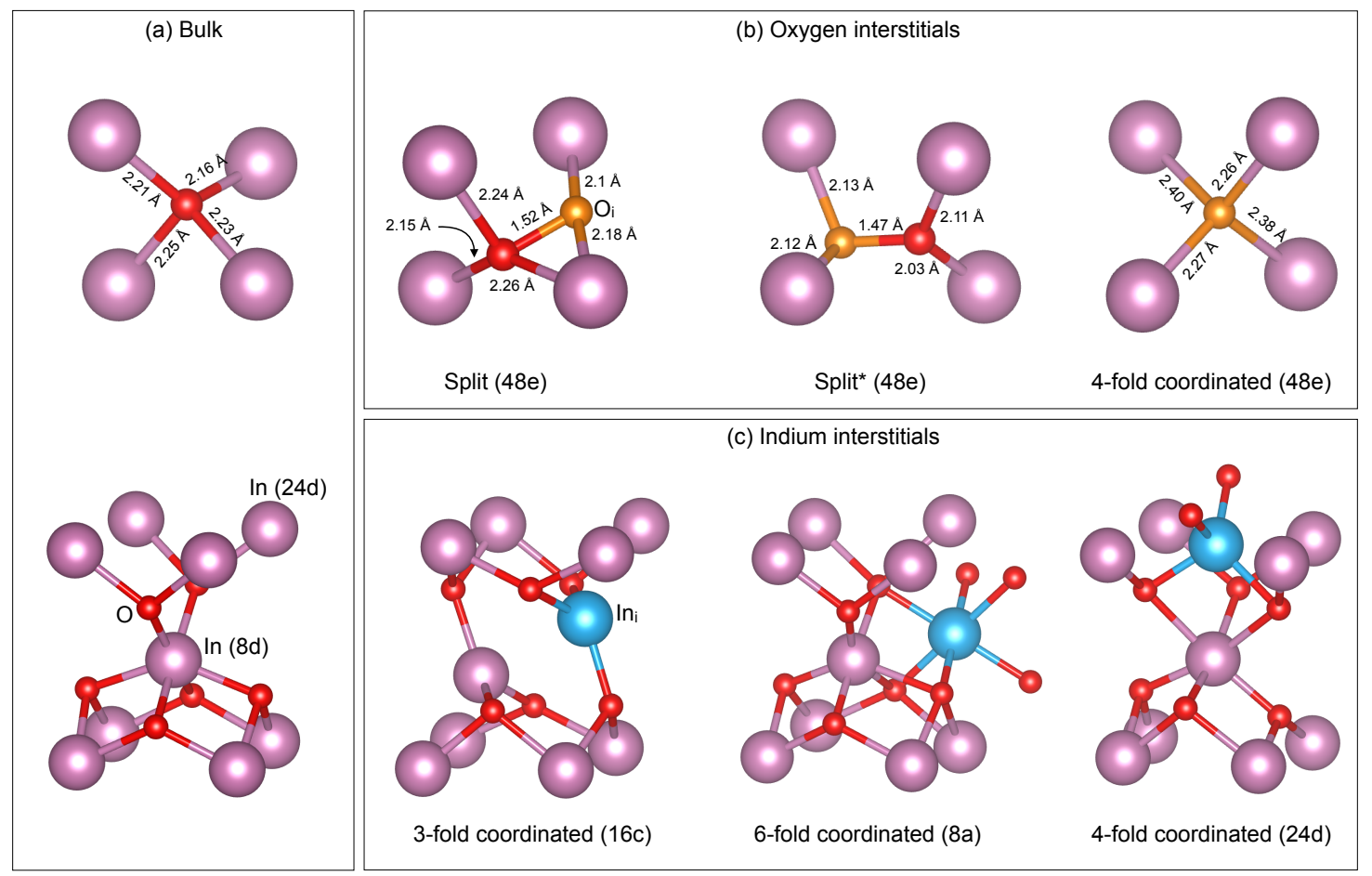

Figure 9: (a) Oxygen site at 48e (top) and Indium sites at $8 \mathrm{~b}$ and 24d (bottom) Wyckoff positions in bulk $\mathrm{In}_{2} \mathrm{O}_{3}$, (b) oxygen interstitials (orange) in split(48e), split*(48e) and 4-fold coordinated (48e), (c) Indium interstitial (blue) in 3-fold (16c), 6-fold (8a), and 4-fold coordinated (24d) configurations.

GGA calculations are performed on 80 atoms supercell, with A $\Gamma$-centered 2x2x2 k-point mesh.

Figure 9 displays the oxygen and indium interstitials structures realized using the automated defect framework. We observe three distinct structures for oxygen interstitials (among the initial set of 22 possible candidates), all occupying the general 48e ( $\mathrm{x}, \mathrm{y}$, z) Wyckoff position in the relaxed structure. Split oxygen interstitial (Fig. 9(b)) is the lowest energy configuration. We find a new split interstitial configuration (referred as split* in Fig. 9(b)) which is stable in the neutral charge state and is about $0.67 \mathrm{eV}$ higher than the lowest energy split interstitial. However split* configuration is unstable in positive charge states and relaxes to the split geometry. Oxygen interstitial bonded to 4 neighboring indium atoms (referred as 4-fold coordinated in Fig. 9(b)) is the highest energy configuration, with energy of about $1.0 \mathrm{eV}$ higher than the split configuration. To our knowledge only the split and 4-fold coordinated oxygen interstitial configuration has been reported in literature [62]. This could be due to the fact that, first, such an exhaustive method to theoretically search interstitials has not been adopted for $\operatorname{In}_{2} \mathrm{O}_{3}$, and second, often only the un-occupied Wyckoff positions (16c and 8 a) are considered to investigate interstitials in $\mathrm{In}_{2} \mathrm{O}_{3}[62]$.

For indium interstitials we also find three distinct configurations (Fig. 9(c)). Lowest energy configuration correspond to indium atom occupying the $16 \mathrm{c}$ $(0.17,0.17,0.17)$ Wyckoff position, which is 3 -fold coordinated to neighboring oxygen atoms (bond length $2.15 \AA$ ). Interstitial atom displaces the indium atom originally at the lattice site $8 \mathrm{~b}$ (with 6 -fold coordination) to a similar $16 \mathrm{c}(0.83,0.17,0.34)$ Wyckoff position with 3 -fold coordination. Indium interstitial 


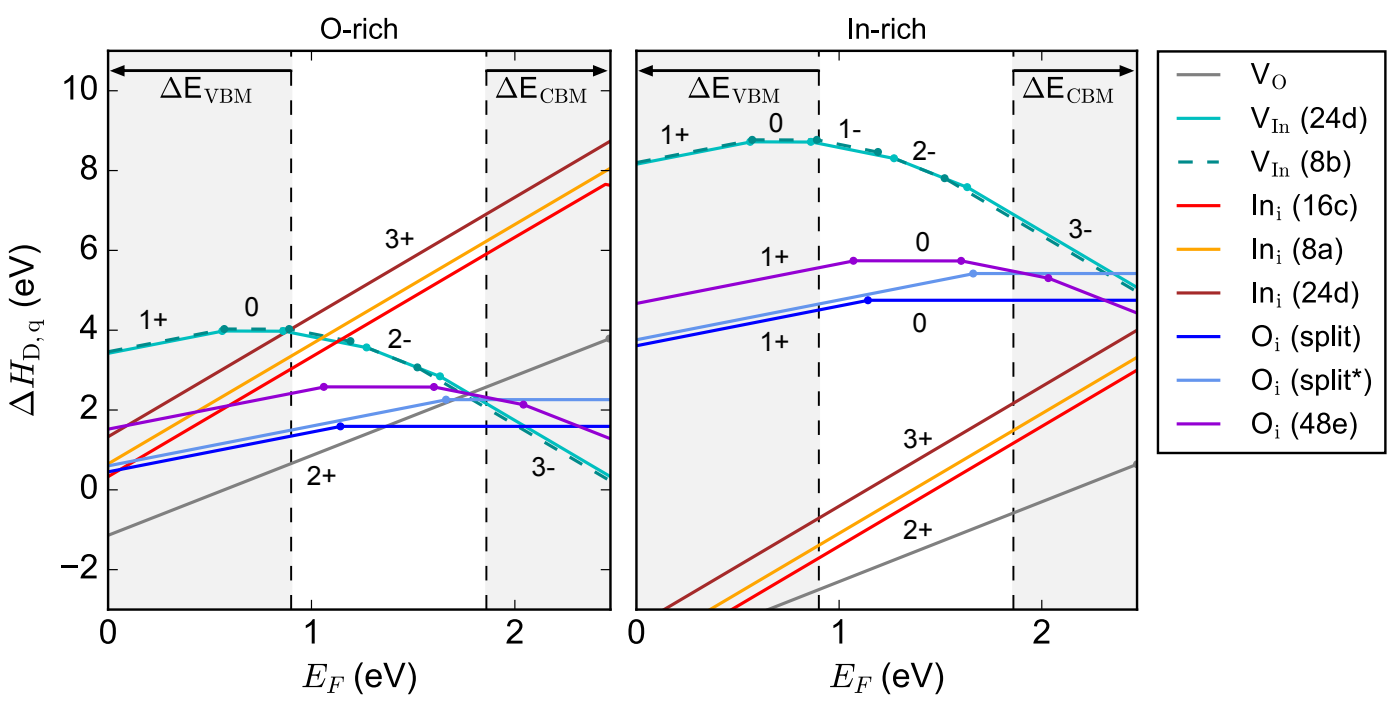

Figure 10: Defect formation energy as function of the Fermi energy for intrinsic vacancies and in $\operatorname{In}_{2} \mathrm{O}_{3}$ using GGA with band edge shifts computed from GW calculation.

at $8 \mathrm{a}(0.5,0.0,0.5)$ Wyckoff position is symmetrically placed in the empty channel between host indium and oxygen atoms along [110] type direction. It is bonded to 6 neighboring oxygen atoms at bond length of 2.24 Å. In neutral charge state its energy is about $0.3 \mathrm{eV}$ higher than 16c configuration. Third indium interstitial configuration occupy the $24 \mathrm{~d}(0.75,0.25,0.50)$ Wyckoff position, and is also placed in the empty channel along [110] type direction. It is bonded to 4 neighboring oxygen atom, two of which are at bond length $2.05 \AA$, and the other two at $2.18 \AA$. $24 \mathrm{~d}$ configuration is highest in energy, with energy $0.83 \mathrm{eV}$ than the 16c configuration in the neutral charge state. To our knowledge, 24d configuration for indium interstitial has never been considered previously, and though it is high in energy, we believe its existence is relevant and crucial because of the entropy at growth temperatures.

In the context of electronic structure, oxygen interstitials has defect states deep inside the band gap, and so as for the indium vacancies (Fig. 10). Indium vacancies have two distinct configurations $8 \mathrm{~b}$ $(0.75,0.25,0.25)$ and $24 \mathrm{~d}(0.75,0.5,0.0)$ both 6fold coordinated to the neighboring oxygen atoms. Indium interstitials in all structural configurations act as shallow donors, with defect states formed as resonance states above the CBM. Indium interstitial occur in $3+$ charge state for the Fermi energy in majority of the band gap, with charge transition levels occur almost at the CBM (Fig. 10). We observe shallow donor type defect states for oxygen vacancy in DFT with $2+$ charge state being the most stable within the explored range of the Fermi energy. As discussed previously [67], defect states in DFT can hybridize strongly with the band edges, and requires self-consistent band gap corrected method such as hybrid functional and defect GW to accurately determine oxygen vacancy charge transition levels. Overall, our results are consistent with previous DFT calculations $[62,66]$ in $\mathrm{In}_{2} \mathrm{O}_{3}$ and demonstrate the potential of the employed automated point defect framework to discover interstitials structures in complex crystal structures.

\section{Summary and future outlook}

We have developed an efficient and extensively validated framework to automate point defect calculations. We applied the framework to $\mathrm{Si}, \mathrm{ZnO}$ and $\mathrm{In}_{2} \mathrm{O}_{3}$, and recovered the known intrinsic defect 
structures as well as their electronic structure properties. Our results demonstrate that the automated defect framework can not only be employed to discover interstitials in complex crystal structures such as $\mathrm{In}_{2} \mathrm{O}_{3}$, but also predict accurate defect formation energy of point defects using the implemented finitesize correction schemes. Regarding application to impurities, the present framework is capable of calculating formation energies of impurities by appropriately taking into account the chemical identities of the impurities. The package is being continuously developed and is hosted on GitHub at https://github. com/pylada/pylada-defects. We believe an automated point defect analysis framework like this will accelerate structure-property prediction by bringing detailed defect understanding to the forefront, and will contribute to more strategic efforts towards tuning the device performance.

\section{Acknowledgments}

We thank Rachel Kurchin for helpful discussions. A. Goyal and V. Stevanovic are funded by the National Science Foundation (NSF) partially under grants DMR-1309980 and CBET-1605495. P. Gorai is funded by the NSF DMR program, grant no. 1334713. H. Peng and S. Lany acknowledges support as part of the Center for the Next Generation of Materials by Design, an Energy Frontier Research Center (EFRC) funded by U.S. Department of Energy (DOE), Office of Science, Basic Energy Sciences. This research used computational resources sponsored by the DOE Office of Energy Efficiency and Renewable Energy and located at the National Renewable Energy Laboratory (NREL).

\section{References}

[1] Jesús A. del Alamo. Nanometre-scale electronics with IIIV compound semiconductors. Nature, 479(7373):317-323, nov 2011.

[2] Xinge $\mathrm{Yu}$, Tobin J. Marks, and Antonio Facchetti. Metal oxides for optoelectronic applications. Nature Materials, 15(4):383-396, 2016.
[3] Jun Yan, Prashun Gorai, Brenden Ortiz, Sam Miller, Scott A. Barnett, Thomas Mason, Vladan Stevanović, and Eric S. Toberer. Material descriptors for predicting thermoelectric performance. Energy Environ. Sci., 8(3):983994, 2015.

[4] Kurt Lejaeghere, Gustav Bihlmayer, T. Bjorkman, Peter Blaha, S. Blugel, Volker Blum, Damien Caliste, Ivano E Castelli, Stewart J Clark, A. Dal Corso, S. de Gironcoli, T. Deutsch, John Kay Dewhurst, I. Di Marco, Claudia Draxl, M. Du ak, Olle Eriksson, J. A. Flores-Livas, Kevin F Garrity, Luigi Genovese, Paolo Giannozzi, Matteo Giantomassi, Stefan Goedecker, Xavier Gonze, O. Granas, E. K. U. Gross, A. Gulans, F. Gygi, D R Hamann, Phil J Hasnip, N A W Holzwarth, D. Iu an, Dominik B Jochym, F. Jollet, Daniel Jones, Georg Kresse, Klaus Koepernik, E. Kucukbenli, Y. O. Kvashnin, Inka L M Locht, Sven Lubeck, Martijn Marsman, Nicola Marzari, Ulrike Nitzsche, L. Nordstrom, Taisuke Ozaki, Lorenzo Paulatto, Chris J Pickard, Ward Poelmans, M. I. J. Probert, K. Refson, M. Richter, G.-M. Rignanese, S. Saha, M. Scheffler, M. Schlipf, K. Schwarz, S. Sharma, F. Tavazza, P. Thunstrom, A. Tkatchenko, M. Torrent, D. Vanderbilt, M. J. van Setten, V. Van Speybroeck, J. M. Wills, J. R. Yates, G.-X. Zhang, and S. Cottenier. Reproducibility in density functional theory calculations of solids. Science, 351(6280):aad3000-aad3000, mar 2016.

[5] Chris G. Van de Walle. First-principles calculations for defects and impurities: Applications to III-nitrides. Journal of Applied Physics, 95(8):3851, 2004.

[6] Audrius Alkauskas, Peter Deák, Jörg Neugebauer, Alfredo Pasquarello, and Chris G. Van de Walle, editors. Advanced Calculations for Defects in Materials. Wiley-VCH Verlag GmbH \& Co. KGaA, Weinheim, Germany, apr 2011.

[7] Christoph Freysoldt, Blazej Grabowski, Tilmann Hickel, Jörg Neugebauer, Georg 
Kresse, Anderson Janotti, and Chris G. Van de Walle. First-principles calculations for point defects in solids. Reviews of Modern Physics, 86(1):253-305, mar 2014.

[8] Chris H. Rycroft. VORO++: A threedimensional Voronoi cell library in $\mathrm{C}++$. Chaos, 19(4), 2009.

[9] Shyue Ping Ong, William Davidson Richards, Anubhav Jain, Geoffroy Hautier, Michael Kocher, Shreyas Cholia, Dan Gunter, Vincent L. Chevrier, Kristin A. Persson, and Gerbrand Ceder. Python Materials Genomics (pymatgen): A robust, open-source python library for materials analysis. Computational Materials Science, 68:314-319, feb 2013.

[10] Stephan Lany and Alex Zunger. Assessment of correction methods for the band-gap problem and for finite-size effects in supercell defect calculations: Case studies for $\mathrm{ZnO}$ and GaAs. Physical Review B, 78(23):235104, dec 2008.

[11] Stephan Lany and Alex Zunger. Accurate prediction of defect properties in density functional supercell calculations. Modelling and Simulation in Materials Science and Engineering, 17(8):084002, dec 2009.

[12] G Makov and MC Payne. Periodic boundary conditions in ab initio calculations. Physical Review B, 51(7):4014-4022, feb 1995.

[13] Christoph Freysoldt, Jörg Neugebauer, and Chris G. Van de Walle. Electrostatic interactions between charged defects in supercells. Physica Status Solidi (B), 248(5):1067-1076, may 2011.

[14] Samuel E. Taylor and Fabien Bruneval. Understanding and correcting the spurious interactions in charged supercells. Physical Review B, 84(7):075155, aug 2011.

[15] Hannu-Pekka Komsa, Tapio T. Rantala, and Alfredo Pasquarello. Finite-size supercell correction schemes for charged defect calculations. Physical Review B, 86(4):045112, jul 2012.
[16] Yu Kumagai and Fumiyasu Oba. Electrostaticsbased finite-size corrections for first-principles point defect calculations. Physical Review B - Condensed Matter and Materials Physics, 89:195205, 2014.

[17] Stephan Lany. Semiconductor thermochemistry in density functional calculations. Physical Review B, 78(24):245207, dec 2008.

[18] Vladan Stevanović, Stephan Lany, Xiuwen Zhang, and Alex Zunger. Correcting density functional theory for accurate predictions of compound enthalpies of formation: Fitted elemental-phase reference energies. Physical Review B, 85(11):115104, 2012.

[19] Lars Hedin. New Method for Calculating the One-Particle Green's Function with Application to the Electron-Gas Problem. Physical Review, 139(3A):A796-A823, aug 1965.

[20] John P. Perdew, Matthias Ernzerhof, and Kieron Burke. Rationale for mixing exact exchange with density functional approximations. The Journal of Chemical Physics, 105(22):9982, 1996.

[21] Jochen Heyd, Gustavo E. Scuseria, and Matthias Ernzerhof. Hybrid functionals based on a screened Coulomb potential. Journal of Chemical Physics, 118(18):8207-8215, 2003.

[22] Haowei Peng, David O. Scanlon, Vladan Stevanovic, Julien Vidal, Graeme W. Watson, and Stephan Lany. Convergence of density and hybrid functional defect calculations for compound semiconductors. Physical Review B, 88(11):115201, sep 2013.

[23] Mayeul d'Avezac, Peter Graf, Tula Paudal, Haowei Peng, Lijun Zhang, Sullivan Stephen, and Vladan Stevanović. Pylada: a comprehensive python framework for preparing, running, monitoring, analyzing, and archiving high throughput first principles calculations. GitHub repository, 2010. 
[24] Wendell Smith. A 3D cell-based Voronoi library based on voro++. GitHub repository, 2015.

[25] Prashun Gorai, Duanfeng Gao, Brenden Ortiz, Sam Miller, Scott A. Barnett, Thomas Mason, Qin Lv, Vladan Stevanović, and Eric S. Toberer. TE Design Lab: A virtual laboratory for thermoelectric material design. Computational Materials Science, 112:368-376, feb 2016.

[26] Vladan Stevanović. Sampling Polymorphs of Ionic Solids using Random Superlattices. Physical Review Letters, 116(7):075503, feb 2016.

[27] Ann M Deml, Ryan O'Hayre, Chris Wolverton, and Vladan Stevanović. Predicting density functional theory total energies and enthalpies of formation of metal-nonmetal compounds by linear regression. Physical Review B, 93(8):085142, feb 2016.

[28] C. R. Hubbard, H. E. Swanson, and F. A. Mauer. A silicon powder diffraction standard reference material. Journal of Applied Crystallography, 8(1):45-48, feb 1975.

[29] O Madelung. Semiconductors: group IV elements and III-V compounds, 1991.

[30] H. Karzel, W. Potzel, M. Köfferlein, W. Schiessl, M. Steiner, U. Hiller, G. M. Kalvius, D. W. Mitchell, T. P. Das, P. Blaha, K. Schwarz, and M. P. Pasternak. Lattice dynamics and hyperfine interactions in $\mathrm{ZnO}$ and $\mathrm{ZnSe}$ at high external pressures. Physical Review B, 53(17):1142511438, may 1996.

[31] N. Ashkenov, B. N. Mbenkum, C. Bundesmann, V. Riede, M. Lorenz, D. Spemann, E. M. Kaidashev, A. Kasic, M. Schubert, M. Grundmann, G. Wagner, H. Neumann, V. Darakchieva, H. Arwin, and B. Monemar. Infrared dielectric functions and phonon modes of highquality $\mathrm{ZnO}$ films. Journal of Applied Physics, 93(1):126, 2003.

[32] O Kubaschewski, P J Spencer, and C B Alcock. Materials thermochemistry, 1993.
[33] U. Ozgur, Ya I. Alivov, C. Liu, A. Teke, M. A. Reshchikov, S. Dogan, V. Avrutin, S.-J. Cho, and H. Morkoc. A comprehensive review of $\mathrm{ZnO}$ materials and devices. Journal of Applied Physics, 98(4):041301, 2005.

[34] J.H.W. De Wit. Structural aspects and defect chemistry in In2O3. Journal of Solid State Chemistry, 20(2):143-148, feb 1977.

[35] I. Hamberg and C. G. Granqvist. Evaporated Sn-doped In2O3 films: Basic optical properties and applications to energy-efficient windows. Journal of Applied Physics, 60(11):R123, 1986.

[36] Martin Feneberg, Jakob Nixdorf, Christian Lidig, Rüdiger Goldhahn, Zbigniew Galazka, Oliver Bierwagen, and James S. Speck. Manyelectron effects on the dielectric function of cubic In2O3 : Effective electron mass, band nonparabolicity, band gap renormalizati. Physical Review B, 93(4):045203, jan 2016.

[37] A Bourlange, D J Payne, R G Egdell, J S Foord, P P Edwards, M O Jones, A Schertel, P J Dobson, J L Hutchison, A Bourlange, D J Payne, R G Egdell, J S Foord, $\mathrm{P}$ P Edwards, and M O Jones. Growth of In2O3 (100) on Y-stabilized ZrO2 (100) by O-plasma assisted molecular beam epitaxy. Applied Physics Letters, 92(092117), 2008.

[38] Aron Walsh, A Klein, L F J Piper, Alex Demasi, Kevin E Smith, Juarez L F Da Silva, Su-huai Wei, C Ko, G Panaccione, P Torelli, D J Payne, A Bourlange, and R G Egdell. Nature of the Band Gap of In 2 O 3 Revealed by First-Principles Calculations and X-Ray Spectroscopy. Physical Review Letters, 100(167402), 2008.

[39] P. D C King, T. D. Veal, F. Fuchs, Ch Y. Wang, D. J. Payne, A. Bourlange, H. Zhang, G. R. Bell, V. Cimalla, O. Ambacher, R. G. Egdell, F. Bechstedt, and C. F. McConville. Band gap, electronic structure, and surface electron accumulation of cubic and rhombohedral In2 O3. Physical 
Review B - Condensed Matter and Materials Physics, 79(20):1-10, 2009.

[40] K. Irmscher, M. Naumann, M. Pietsch, Z. Galazka, R. Uecker, T. Schulz, R. Schewski, M. Albrecht, and R. Fornari. On the nature and temperature dependence of the fundamental band gap of In2O3. physica status solidi (a), 211(1):54-58, jan 2014.

[41] G. D. Watkins. Native Defects and their Interactions with Impurities in Silicon. MRS Proceedings, 469(9):139, jan 1997.

[42] H. Bracht, H. H. Silvestri, I. D. Sharp, and E. E. Haller. Self- and foreign-atom diffusion in semiconductor isotope heterostructures. II. Experimental results for silicon. Physical Review B - Condensed Matter and Materials Physics, 75(3):1-21, 2007.

[43] G. A. Baraff, E. O. Kane, and M. Schlüter. Silicon Vacancy: A Possible "Anderson Negative- U" System. Physical Review Letters, 43(13):956-959, sep 1979.

[44] Roberto Car, Paul J. Kelly, Atsushi Oshiyama, and Sokrates T. Pantelides. Microscopic Theory of Atomic Diffusion Mechanisms in Silicon. Physical Review Letters, 52(20):1814-1817, may 1984.

[45] Y. Bar-Yam and J. D. Joannopoulos. Barrier to Migration of the Silicon Self-Interstitial. Physical Review Letters, 52(13):1129-1132, mar 1984.

[46] M. Puska, S. Pöykkö, M. Pesola, and R. Nieminen. Convergence of supercell calculations for point defects in semiconductors: Vacancy in silicon. Physical Review B, 58(3):1318-1325, 1998.

[47] A. F. Wright. Density-functional-theory calculations for the silicon vacancy. Physical Review B, 74(16):165116, oct 2006.

[48] Patrick Rinke, Anderson Janotti, Matthias Scheffler, and Chris G. Van De Walle. Defect formation energies without the band-gap problem: Combining density-functional theory and the GW approach for the silicon self-interstitial. Physical Review Letters, 102(2):1-4, 2009.

[49] Piotr Śpiewak and Krzysztof J. Kurzydłowski. Formation and migration energies of the vacancy in Si calculated using the HSE06 rangeseparated hybrid functional. Physical Review B, 88(19):195204, nov 2013

[50] P. E. Blöchl. Projector augmented-wave method. Physical Review B, 50(24):17953-17979, dec 1994.

[51] G Kresse and J. Furthmüller. Efficiency of abinitio total energy calculations for metals and semiconductors using a plane-wave basis set. Computational Materials Science, 6(1):15-50, jul 1996.

[52] Jp Perdew, K Burke, and M Ernzerhof. Generalized Gradient Approximation Made Simple. Physical review letters, 77(18):3865-3868, oct 1996.

[53] Hendrik J. Monkhorst and James D. Pack. Special points for Brillouin-zone integrations. Physical Review B, 13(12):5188-5192, jun 1976.

[54] Alec Belsky, Mariette Hellenbrandt, Vicky Lynn Karen, and Peter Luksch. New developments in the Inorganic Crystal Structure Database (ICSD): accessibility in support of materials research and design. Acta Crystallographica Section B Structural Science, 58(3):364-369, jun 2002.

[55] Aliaksandr V. Krukau, Oleg A. Vydrov, Artur F. Izmaylov, and Gustavo E. Scuseria. Influence of the exchange screening parameter on the performance of screened hybrid functionals. The Journal of Chemical Physics, 125(22):224106, 2006.

[56] Fabiano Corsetti and Arash A. Mostofi. Systemsize convergence of point defect properties: The case of the silicon vacancy. Physical Review B, 84(3):035209, jul 2011. 
[57] J. R. Weber, A. Janotti, and C. G. Van de Walle. Dangling bonds and vacancies in germanium. Physical Review B, 87(3):035203, jan 2013.

[58] Wei Chen and Alfredo Pasquarello. Band-edge positions in GW: Effects of starting point and self-consistency. Physical Review B - Condensed Matter and Materials Physics, 90(16):1-15, 2014.

[59] Anderson Janotti and Chris G. Van De Walle. Native point defects in ZnO. Physical Review B - Condensed Matter and Materials Physics, 76(16):1-22, 2007.

[60] Gui Yang Huang, Chong Y. Wang, and Jian T. Wang. First-principles study of diffusion of zinc vacancies and interstitials in $\mathrm{ZnO}$. Solid State Communications, 149(5-6):199-204, 2009.

[61] P Erhart and K Albe. First-principles study of migration mechanisms and diffusion of oxygen in zinc oxide. Physical Review B, 73(11):115207, 2006 .

[62] Stephan Lany and Alex Zunger. Dopability, intrinsic conductivity, and nonstoichiometry of transparent conducting oxides. Physical Review Letters, 98(4):2-5, 2007.

[63] Fumiyasu Oba, Atsushi Togo, Isao Tanaka, Joachim Paier, and Georg Kresse. Defect energetics in $\mathrm{ZnO}$ : A hybrid Hartree-Fock density functional study. Physical Review B - Condensed Matter and Materials Physics, $77(24): 3-8,2008$.

[64] J.H.W. De Wit, G. Van Unen, and M. Lahey. Electron concentration and mobility in $\mathrm{In} 2 \mathrm{O} 3$. Journal of Physics and Chemistry of Solids, 38(8):819-824, jan 1977.

[65] Péter Ágoston, Karsten Albe, Risto M. Nieminen, and Martti J. Puska. Intrinsic n-type behavior in transparent conducting oxides: A comparative hybrid-functional study of In2O3, $\mathrm{SnO} 2$, and $\mathrm{ZnO}$. Physical Review Letters, 103(24):245501, dec 2009.
[66] Jian Liu, Tingyu Liu, Fengming Liu, and Haixin $\mathrm{Li}$. Thermodynamics of native defects in In2O3 crystals using a first-principles method. RSC Advances, 4(70):36983, 2014.

[67] Stephan Lany and Alex Zunger. Comment on intrinsic n-type behavior in transparent conducting oxides: A comparative hybrid-functional study of In2O3, SnO2 and $\mathrm{ZnO}$. Physical Review Letters, 106(069601), 2011. 